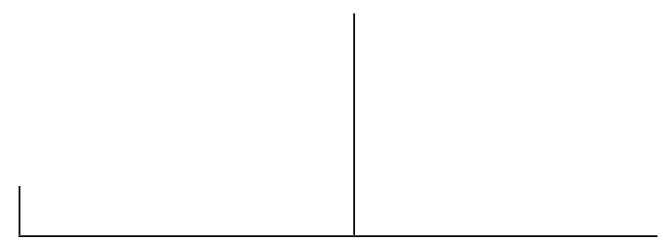

Rev. Latinoam. Psicopat. Fund., São Paulo, 16(1), 126-146, mar. 2013

\title{
O desenvolvimento inicial das ideias de Kraepelin sobre classificação: uma história conceitual*
}

\author{
German E. Berrios \\ R. Hauser
}

A Psiquiatria ainda vive em um mundo kraepeliniano e os estudos sobre a obra de Kraepelin são, por conseguinte, ahistóricos. A exegese seletiva das várias edições de seu tratado levaram a uma visão rígida sobre sua contribuição. No entanto, Kraepelin viveu e escreveu durante um período importante da história intelectual da Europa e sua obra só pode ser entendida neste contexto. Este artigo analisa o desenvolvimento de suas opiniões em termos do "Programa de Pesquisas" que ele planejou precocemente em sua vida e cujo objetivo era a criação de uma descrição estável e da classificação das psicoses. Isso Kraepelin eventualmente alcançou estudando longitudinalmente coortes de pacientes em termos de critérios metodológicos, tais como o curso da doença e a incurabilidade. No caso, esta metodologia permitiu-lhe identificar por correlação "quadros clínicos" que tanto representavam a "essência" da doença quanto forneciam um critério taxonómico. Embora declaradamente ateórico, Kraepelin assim conseguiu construir (influenciado por Kahlbaum e por Wundt) um suporte empírico para sua categorização kantiana das psicoses. Uma discussão sobre as variáveis culturais que moldaram essas ideias está aqui incluída.

Palavras-chave: Transtornos mentais - história, transtornos mentais -classificação, psiquiatria - história, Kraepelin, Emil

* Publicação original: The early development of Kraepelin's ideas on classification: a conceptual history. Psychological Medicine, 18(4), 813-821, 1988. Tradução de Lazslo A. Ávila e revisão técnica de Ana Maria G. R. Oda. 


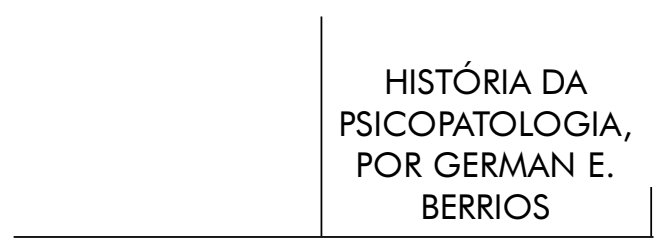

Introdução

A investigação acadêmica acerca de Emil Kraepelin (1856-1926) passou por três fases: a de obituários (Lange, 1926; Wirth, 1927); os escritos em torno do centenário do seu nascimento (Mayer, 1956; Braceland, 1956; Kolle, 1957; Kahn, 1956; Havens, 1956; Wender, 1963; Líder, 1968; Bruch, 1969), e o debate em Londres sobre o que Kraepelin "realmente disse" (Hoenig, 1968; Fish, 1967, 1968; Walk, 1968). Estes, e de fato muitas das publicações posteriores (Rieder, 1974; Blashfield, 1984; Hoff, 1985), têm sido o trabalho de clínicos, daí, eles tendem a ser hagiográficos no tom e exegéticos no estilo.

Os estudos kraepelinianos ganharam um sentido de urgência na década de 1970, particularmente nos EUA, após o eclipse do bleulerianismo e a redescoberta de Kraepelin (Blashfield, 1982; Zubin, 1987). O trabalho histórico subsequente tem, no entanto, sido de qualidade indiferente (por exemplo, Rieder, 1974; Blashfield, 1984), sua principal desvantagem sendo um tratamento a-histórico de Kraepelin. A dicotomia kraepeliniana (da demência precoce e da loucura maníaco-depressiva) continua a ser consagrada no pensamento psiquiátrico e, com poucas exceções, controla as pesquisas neuroquímicas e genéticas. Taxonomias alternativas, como a de Wernicke (1848-1905) ou de Magnan (1835-1916) foram levadas a cair em desuso. A psiquiatria ainda vive em um mundo kraepeliniano e seus praticantes não podem escapar da ofuscante adoção de sua "episteme".

Uma análise histórica das ideias de Kraepelin deve ajudar o médico a ganhar alguma perspectiva. Infelizmente, os historiadores sociais da psiquiatria têm pouco gosto por histórias de casos detalhados e parecem aceitar a visão de que, para Kraepelin, "uma pessoa mentalmente doente parece ser um conjunto de sintomas" (Zilboorg, 1941, p. 452; Decker, 1977). Essa visão tem sido ecoada até por historiadores simpáticos: "o que se sente faltar na descrição de Kraepelin do quadro clínico é a 'psique'. Os sintomas seriam sintomas da doença subjacente. A história de vida do paciente, sua personalidade pré-mórbida, e mesmo sua própria 
experiência da doença não tinham lugar atribuído no esquema das coisas. Eles seriam uma irrelevância" (Hoenig, 1983, p. 549).

Esta visão é com toda a probabilidade imprecisa e não coincide com as preocupações reais de Kraepelin (1920):

(...) em que medida e por quais métodos clínicos podemos compreender mais claramente as manifestações da loucura? os sintomas e sinais que correspondem aos da doença subjacente são extraordinariamente variados. Isto implica que as condições antecedentes devem ter sido complexas. Mesmo quando claros agentes externos estão envolvidos (por exemplo, um ferimento na cabeça ou envenenamento) (...) há um jogo de forças em ação: o sistema nervoso do indivíduo afetado, os déficits herdados das gerações passadas e sua própria história pessoal (...) estas condições são especialmente importantes quando se considera as formas da doença que não surgem de lesões externas, mas das circunstâncias da pessoa em causa (...) parece absurdo propor que seja a sífilis que faça com que os pacientes acreditem que são os possuidores orgulhosos de carros (...) ao invés de que sejam os desejos usuais dessas pessoas refletidos nesses delírios (...) Se essas observações se aproximam da verdade teremos que buscar a chave para a compreensão do quadro clínico, principalmente nas características do paciente individual (...) suas expectativas desempenham um papel decisivo. ( $\mathrm{pp} 2$ -3 , tradução livre; grifos adicionais) ${ }^{2}$

Este equívoco resultou provavemente de uma leitura seletiva da obra de Kraepelin. Por exemplo, pouco se fala dos mais de sessenta artigos que ele publicou após 1913, quando apareceu a oitava edição do seu livro (que se pensa conter todos magister dicta). Esta negligência é surpreendente, pois Kraepelin se aposentou apenas em 1922 e a produção de seus anos mais maduros inclui trabalho conceitual de grande profundidade e beleza. Ele até expressou dúvidas sobre o valor clínico da dicotomia anteriormente formulada (Kraepelin, 1920). Por isso, é salutar que um notável historiador da psicanálise tenha sentido a necessidade de escrever:

Kraepelin se tornou o bode expiatório de muitos psiquiatras atuais que afirmam que sua única preocupação era colocar rótulos diagnósticos em seus pacientes, após o que nada mais seria feito para ajudá-los. Na verdade, porém, ele tomou o maior cuidado para que cada um de seus pacientes pudessem ter o melhor tratamento disponível no momento (...) (Ellenberger, 1970, p. 285)

Este artigo procura abrir um espaço de investigação, atentando para a formação das ideias de Kraepelin sobre a classificação. Já que há consenso de que

\footnotetext{
${ }^{1}$ Todas as traduções (para o inglês), são dos autores.
} 


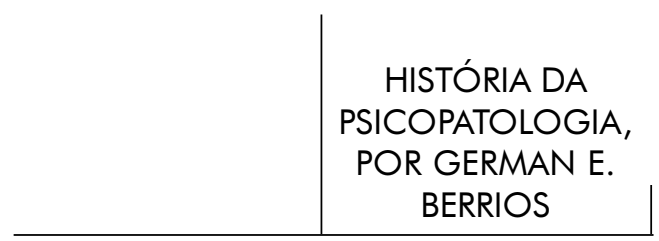

uma de suas contribuições foi o uso que fez do conceito de "prognóstico" como um critério metodológico, este artigo irá explorar o contexto histórico em que essas ideias se desenvolveram e testar a visão de que a observação clínica desempenhou um papel crucial na sua criação, ou seja, de que para ele "a teoria era apenas a serviçal da práxis" (Avenarius, 1979, p. 63). Irá fazê-lo buscando a correspondência das expectativas teóricas de Kraepelin contra os resultados do "Programa de Pesquisa" que ele delineou para si próprio no início de sua carreira.

\section{O contexto sociopolítico}

Kraepelin cresceu durante um dos períodos mais importantes da história alemã e os primeiros quarenta anos de sua vida se desenvolveram no mundo bismarckiano. Ele testemunhou a estratégia de longo prazo do Chanceler de Ferro, que levou à libertação da Prússia da influência austríaca e à unificação dos reinos alemães. Kraepelin tinha apenas 15 anos quando Bismarck se tornou chanceler e os eventos como a derrota da França (e os excessos perpetrados pelo exército prussiano) e o ataque persistente à Igreja Católica Romana pelos liberais prussianos o afetaram profundamente. Ele ainda estava na escola em Neustrelitz quando Rudolf Virchow (um de seus heróis), um deputado da esquerda liberal, cunhou o termo "Kulturkampf" em um famoso discurso no qual afirmou que a luta contra a Igreja Romana asseguraria "o caráter de uma grande luta pela civilização, no interesse da humanidade" (Craig, 1978). Kraepelin era politicamente conservador e tinha grande admiração por Bismarck (Alexander \& Selesnick, 1966). Isto é corroborado por seu longo artigo "A personalidade de Bismarck", onde ele tentou estabelecer um paralelo entre o perfil psicológico do chanceler alemão e o dele próprio (Kraepelin, 1921).

A política bismarckiana invadia até mesmo o debate científico. A luta entre os localizacionistas (por exemplo, Hitzig e Fritsch) e seus opositores ocorreu em tal contexto. O sucesso dos primeiros deveu-se menos à sua força científica (o "experimento crucial" ainda não era possível no momento) do que ao fato de que ela refletia o rigor da cultura bismarckiana. Como Pauly (1983) afirmou:

(...) a localização tanto representava a ordem burocrática quanto era um meio para promover a ordem de forma concreta. Ela deu apoio crucial para o argumento de que as doenças mentais eram as doenças do cérebro, trazendo assim a insanidade mais firmemente para dentro dos limites da medicina (...) (p. 146)

Kraepelin foi um localizacionista convicto e dependeu do sucesso desta teoria. Assim, ele prestou homenagem a Hitzig em seu Cem anos de psiquiatria 
(Kraepelin, 1918) e escreveu com admiração sobre Gudden (Kraepelin, 1983, pp. 16-17). Sua carreira foi caracterizada por um constante progresso, e, aparte seu embate com Flechsig no verão de 1882 (que levou à sua demissão de um emprego promissor), ele parece ter jogado suas cartas políticas com sucesso.

\section{Dorpat e a aula inaugural}

Em sua ascensão para a cadeira de psiquiatria na Universidade de Dorpat, Kraepelin proferiu a aula inaugural em 1886 . Na época, ele tinha trinta anos e estava trabalhando em psiquiatria há apenas oito anos (incluindo um período fazendo psicologia experimental sob orientação de Wundt). Ele substituiu Emminghaus (1845-1904), um velho amigo e protetor, que mais tarde iria ajudá-lo a obter a cadeira de Heidelberg.

A conferência foi intitulada: "As direções da pesquisa psiquiátrica" (Kraepelin, 1887). A psiquiatria acadêmica e institucional (ou asilar) estavam no momento empenhadas em luta feroz (Jaspers, 1963, pp. 846-847) e o título de sua conferência deixava claro de que lado ele estava. Ele anunciou sua intenção de combinar pesquisa e cuidados ao paciente psiquiátrico e definiu os objetivos para a primeira: no curto prazo, ele iria procurar por grupos válidos de doenças e, a longo prazo, procuraria as "leis" que ligam os dados anatômicos aos psicológicos. No que diz respeito à formação de grupos de doenças, ele pensava que, na ausência de critérios neuropatológicos, teria de se contentar com as descrições clínicas. Ele tinha percebido isso tão cedo quanto 1879, quando, após a realização de muitas dissecações do cérebro com Ganser e Bumm, ele observou: “(...) não fomos ainda capazes de diferenciar entre o córtex de um paciente com $\mathrm{Pa}$ ralisia Geral e o de um sujeito normal, e muito menos identificar a doença por suas características patológicas" (Kraepelin, 1983, p. 18).

Em sua busca por obter descrições estáveis de doença ele recorreu primeiro a definições operacionais: "Tentei organizar as manifestações confusas da doença em meus pacientes, descrevendo o seu comportamento tão exatamente quanto possível..." (Kraepelin, 1983, p. 35). Ele expressou insatisfação com a classificação contemporânea, porque a sua "taxa"* não era distinta o bastante, mas citou Karl Kahlbaum (1826-1899) com aprovação, já que este autor tinha levantado

* Taxa: plural de táxon, em biologia, unidade de um sistema de classificação (classe, ordem, família, gênero, espécie etc.). ( $\mathrm{N}$ da $\mathrm{R}$.). 


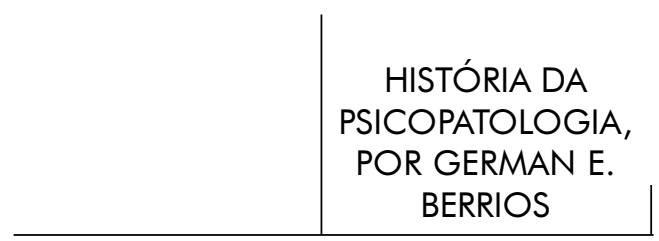

críticas semelhantes. Em Dorpat, ele também "foi levado a considerar a importância do curso da doença no que diz respeito à classificação das perturbações mentais" (Kraepelin, 1983, p. 49). Esta intenção declarada de desenvolver uma classificação, expressa já em 1887, contraria a alegação de pelo menos um comentarista: "Kraepelin, pelo menos originalmente, não tinha a intenção de criar novas classificações" (Blashfield, 1984, p. 11I).

\section{A influência de Kahlbaum sobre Kraepelin}

Kahlbaum (1863) havia proposto uma "abordagem empírico-clínica" para a classificação em seu muito citado (embora raramente lido) livro. Ali, ele também enfatizou a importância do "curso da doença" e fez uma distinção entre os conceitos transversal (sincrônico) e longitudinal (diacrônico) de doença. A "essência" da doença (e não está claro como era a importação "ontológica" de seu uso da palavra "essência") se encontrava em sua história natural e perfil temporal. Não se deve esquecer que, até então, "tempo" (a dimensão temporal) não fazia parte da definição de doença psiquiátrica (Lanteri-Laura, 1972; Del Pistoia, 1971). Kahlbaum sugeriu a distinção entre estados agudos e crônicos. Isto só iria se cristalizar no conceito francês de "délire chronique" (Lanteri-Laura, 1986) e na obra do próprio Kraepelin.

Os pontos de vista de Kahlbaum em 1863 eram, no entanto, abstratos e pouco continham de suporte empírico. Kraepelin buscou testá-los, perguntando que "aspectos" (ou sintomas) forneciam informação no que diz respeito à "essência" de uma doença. Ele esperava que, uma vez identificados, esses sintomas poderiam ser utilizados como critérios para gerar uma classificação que fosse, pela primeira vez, baseada in re. Ele colocou de forma sucinta:

É verdade que, neste momento ainda não podemos encontrar uma saída do labirinto de quadros clínicos. Nossa situação atual é que somos incapazes de escolher dentre um vasto número os elementos ou sinais que podem ser essenciais ou característicos no caso concreto. (Kraepelin, 1887, pp. 20-21)

E eu pensei que poderia ser possível encontrar cursos similares de doença e doenças uniformes pela observação retrospectiva (...) (Kraepelin, 1983, p. 49)

Para resumir, por volta de 1886, a questão central para Kraepelin era encontrar sinais "essenciais" de doença sobre os quais basear tanto o diagnóstico quanto a classificação. 


\section{A perspectiva clínica de Kraepelin}

Dez anos mais tarde, Kraepelin (1896) anunciou que tinha "encontrado uma nova maneira de olhar para a doença mental". Os termos que ele usou para se referir às abordagens tradicional e nova eram "sintomática" e "clínica", respectivamente.

Em Dorpat, Kraepelin também era diretor da Clínica Universitária, de oitenta leitos, e ali começou a prestar atenção ao conceito de "curso da doença". Os regulamentos russos na cidade permitiam a admissão de pacientes "informais" ou voluntários, em contraste com a legislação alemã que dizia que todos os pacientes tinham de estar sob uma seção ("bezirksaerztliche Einweisung”). Isto enviesava a população de pacientes de Dorpat na direção de casos mais leves e mais recentes e, acima de tudo, reduzia a alta proporção de transtornos mentais orgânicos agudos e crônicos, uma das maldições das populações asilares do século XIX. Com Daraszkiewicz ele estudou detalhadamente os históricos clínicos. Sua primeira observação foi que a dilapidação psicológica era comum, mas adquirida (ou seja, tinha aparecido de novo [recentemente] durante a doença). Isto levou-os a postular a presença de um processo degenerativo (Kraepelin, 1983). Tem sido sugerido que este conceito de deterioração foi obtido diretamente da "teoria da degeneração" ainda ativa (Zubin et al., 1985). Isso pode ser tomado no sentido muito geral de que todos os psiquiatras do final do século XIX tinham de tomar uma posição vis-à-vis alguma variante desta ideia, mas não deve ser superestimada. Este artigo apresenta evidências que sugerem que Kraepelin teve uma abordagem mais empírica para este assunto. Neste sentido, dado o fato de que a noção de demência como um estado "adquirido" ainda não tinha sido totalmente cristalizada naquela época, sua conclusão empírica foi uma observação importante (Berrios, 1987a).

Uma variável cultural adicional estava em operação. A clínica Dorpat (Tartu na atual Estônia) servia a uma comunidade de não falantes da língua alemã, cujas principais línguas eram o Dorpat (ou Werro-Estoniano) e o Reval-Estoniano. O primeiro, o mais comum dos dois, era também o menos literário e inflexional (Anônimo, 1878). Apenas um treze avos (1/13) da população falava alemão, mas este grupo pertencia às classes superiores, que eram susceptíveis de procurar tratamento médico na Alemanha. Kraepelin, por conseguinte, teve de utilizar um intérprete para examinar a maioria dos seus pacientes (Kraepelin, 1983). É provável que esta barreira linguística tenha contribuído para a criação de seu interesse de longa duração nos sinais comportamentais (por exemplo, psicomotores) da insanidade. 
HISTÓRIA DA

PSICOPATOLOGIA,

POR GERMAN E.

BERRIOS

A distinção entre a "forma" (ou "essência") de uma doença e suas diversas apresentações (fenômenos) havia sido introduzida por Kahlbaum em 1863. Kraepelin (1983) sabia do assunto pelo menos desde 1879. Durante este período, ele ainda era crítico dos conceitos de Kahlbaum (ele permaneceu assim até 1889), alegando que esses eram demasiado abstratos e poderiam levar a uma forma de psicose unitária, ou seja, à visão de que todos os estados clínicos seriam manifestações de uma única doença.

Por volta de 1889 , Kraepelin parece ter abandonado essa interpretação estreita dos pontos de vista de Kahlbaum. Não há informação direta disponível sobre as razões ou modo como isso se deu. Daraszkiewicz, conhecido por ter sido um assistente brilhante e como autor de uma monografia excelente sobre a hebefrenia (1892), era muito a favor de Kahlbaum e Hecker (1843-1909) (seguidor de Kahlbaum), e seu entusiasmo pode muito bem ter influenciado Kraepelin. Na verdade, este último lhe dá crédito por haver atraído a sua atenção nesse sentido.

\section{O período de Heidelberg}

Em 1891, Kraepelin foi nomeado para a cátedra de Heidelberg. Ele também assumiu uma clínica psiquiátrica bem organizada (construída em 1878) e um laboratório neuropatológico. Ele poderia, assim, passar mais tempo em pesquisa. O edifício estava perto de uma estação ferroviária, e isto facilitava a presença dos pacientes e seu acompanhamento. Kraepelin decidiu que era hora de implementar o método longitudinal de Kahlbaum. Ele também estava cercado por uma grande equipe acadêmica e isso permitiu-lhe seguir a orientação de Kahlbaum de que monografias especializadas eram essenciais para estimular o crescimento em determinadas áreas. Na verdade, ele falou com entusiasmo sobre o papel desse gênero de estudo na reunião de Karlsruhe em 1892 (Kraepelin, 1894).

Assim, Kraepelin pôde colocar em prática o que era só um programa teórico nos escritos de Kahlbaum. Ele começou um "Programa de pesquisa" (a frase é de Kraepelin) (Kraepelin, 1897) cujo objetivo era seguir uma coorte de pacientes e analisar estatisticamente os resultados. O programa começou em 1891 e levou a uma coleção sistemática de todos os casos, evitando assim o viés de incluir apenas os casos "interessantes" (Kraepelin, 1919; Grayson, 1987). Dentro de quatro semanas os pacientes admitidos recebiam um diagnóstico e um prognóstico (em notas separadas). Os contatos de acompanhamento eram ativamente prosseguidos no ambulatório e, no caso de pacientes de longa estadia, nos próprios asilos locais. Notas clínicas eram coletadas em fichas de registro (index cards) especialmente delineadas (as Zählkarten). 
Kraepelin teve de lidar com o fato de que, em Heidelberg, as amostras de pacientes eram enviesadas na direção da gravidade e cronicidade. Há três razões para isso: os pacientes em Baden só podiam ser admitidos em uma seção, a Clínica não podia recusar admissões, e havia uma tendência para o acúmulo de pacientes, pois os hospitais de longa permanência em Pforzheim e Emmendingen (aos quais os pacientes poderiam, em teoria, ser transferidos) estavam sempre ocupados. Para resolver este problema, Kraepelin sugeriu três alterações ao ministro encarregado: a construção de outro hospital de longa permanência, o direito da Clínica selecionar pacientes, e a aceitação da admissão voluntária. Por isso, ele se opôs a psiquiatras "institucionais" (por exemplo Schuele, de Illenau) que queriam desviar fundos para os asilos e reduzir o poder dos departamentos universitários. Kraepelin não teve sucesso em sua oferta e, como resultado, os pacientes crônicos continuaram a ocupar seus leitos. Essa dificuldade pode ter contribuído para sua decisão de deixar Heidelberg (Janzarik, 1979). Entretanto, a resposta de Kraepelin foi construtiva e levou-o a explorar mais os problemas da deterioração e prejuízos.

\section{Critérios de Kraepelin}

Seus dados sugeriram-lhe que uma grande proporção de pacientes, fosse qual fosse a apresentação clínica anterior, acabavam em estado de demência. Ele colocou assim:

Gradualmente me dei conta de que muitos pacientes, que inicialmente apresentavam um quadro de mania, melancolia ou amência mostravam demência progressiva. Apesar das diferenças individuais eles começavam a se parecer uns com os outros. Era como se as diferenças clínicas anteriores tivessem pouca influência sobre o decurso da doença. Esta evolução era semelhante ao que já era conhecido no que diz respeito à paralisia. Assim, não pude resistir a concluir que apenas um processo de doença poderia estar afetando muitos dos doentes institucionalizados que desenvolveram demência. O processo pode ser lento ou rápido e, por vezes acompanhado por delírios, alucinações e excitação. Em algumas ocasiões, pode haver um estado de humor triste (ou exaltado) - qualquer que seja sua apresentação, sempre conduzindo à destruição da personalidade. (Kraepelin, 1983)*

\footnotetext{
* Todas as citações sem indicação de página, estão dessa forma no original. (N. da R.)
} 


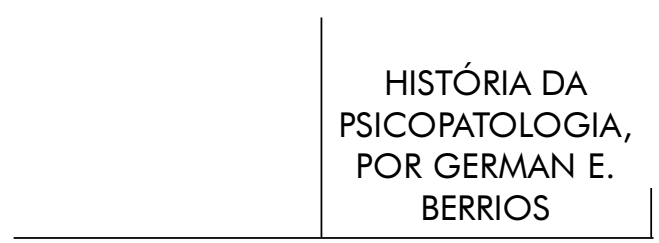

Tendo chegado a esta conclusão, Kraepelin procurou identificar os indicadores iniciais para o processo degenerativo. Ou seja, ele usou a deterioração como um critério de pesquisa ou metodológico para procurar um quadro de predição clínica que, por sua vez, tornar-se-ia um critério clínico. De 1893 em diante, ele simplificou o processo de acompanhamento (Kraepelin, 1897, 1983) e o diagnóstico e o prognóstico foram inseridos nas fichas de registro logo após a primeira entrevista. $\mathrm{O}$ objetivo da coleta de informações na mesma ficha era facilitar a busca de correlações entre a apresentação precoce e as alterações posteriores.

Kraepelin desenvolveu seus pontos de vista sobre o prognóstico ainda mais. Isto pode ser mapeado nas sucessivas edições do Lehrbuch e nas palestras que ele deu sobre o tema. Seus pontos de vista geraram um debate acalorado na época (veja abaixo). Os primeiros resultados de seu programa de pesquisa permitiram a Kraepelin moldar dois conceitos clínicos: "processo degenerativo psicológico" e "demência precoce" (não há espaço aqui para relacionar este último termo ao termo francês démence précoces. Estes termos, introduzidos na quarta edição (1893) do Lehrbuch, foram definidos assim:

A característica comum dessas doenças que agrupei sob o nome de processos de degeneração psicológica é o rápido desenvolvimento de um estado duradouro de fraqueza psicológica... (p. 435). O que chamamos de demência precoce é o desenvolvimento subagudo de uma condição peculiar e simples de fraqueza mental que ocorre em uma idade juvenil. (Kraepelin, 1893, p. 435)

Em um artigo sobre "Remissão em catatonia", lido em uma reunião da Sociedade de Psiquiatria, em novembro de 1895, Kraepelin (1896a) criticou os pontos de vista de Kahlbaum e outros sobre o prognóstico relativamente bom da catatonia. Kraepelin relatou uma coorte de 63 casos em que, dos 24 que haviam melhorado, 14 tinham sido readmitidos dentro de cinco anos. Mais importante, esses 24 pacientes não tinham ficado livres de sintomas durante o intervalo (ele apresentou um acompanhamento detalhado de dez casos).

Em março de 1896, Kraepelin publicou a importante quinta edição do Lehrbuch. Nela ele descreveu seu método para o registro dos dados clínicos:

O que me convenceu da superioridade do método clínico de diagnóstico (seguido aqui) sobre o tradicional, foi a certeza com que podíamos prever (em conjunto com nosso novo conceito de doença) o curso futuro dos eventos [grifo no original]. Graças a ele o aluno pode encontrar o seu caminho com mais facilidade na difícil disciplina da psiquiatria. (Kraepelin, 1896b)

Nesta fase, portanto, Kraepelin sentiu que tinha conseguido um de seus objetivos e afirmou isso na reunião de Heidelberg em 1896 da Associação dos Psiquiatras Alemães (Kraepelin, 1897). Em seu artigo ele ressaltou a importância 
de fazer diagnósticos "válidos", e depois de fazer retoricamente a "pergunta Dorpat" (isto é, como diferenciar os sintomas essenciais dos acidentais), ele afirmou que o único critério externo disponível para classificação dos sintomas tinha de ser o curso da doença. A observação longitudinal estrita e a apuração do estado terminal permitem ao clínico diferenciar entre os sintomas essenciais e os acidentais (Kraepelin, 1897).

Ele também relatou os resultados de seu trabalho correlacional, com base nas fichas de acompanhamento, e ainda listou dois grupos de sintomas: os comuns a todas as psicoses, mas incapazes de prever o resultado, como os "enganos sensoriais", alucinações e patologias afetivas, e aqueles presentes somente em algumas psicoses, mas capazes de prever a deterioração, como a "fuga de ideias, falhas de orientação, perturbações das percepções, excitação e inibição motora". (Kraepelin, 1897, p. 841). Este ponto de vista de que apenas alguns sintomas trazem informações sobre o curso da doença, é ainda vivo na psiquiatria corrente, particularmente em relação aos chamados sintomas positivos e negativos (Berrios, 1985, 1987b).

Contra os pontos de vista de Kraepelin, reuniu-se uma oposição considerável de homens como Meschede, Siemerling, Mendel, Grashey e Jolly. O último (1844-1904), ex-editor da Archiv für Psychiatrie e um alienista respeitado em Berlim, criticou o uso idiossincrático de Kraepelin do prognóstico como uma chave para o diagnóstico e declarou que isso "contradizia os princípios gerais da patologia". Ele assinalou o ponto interessante de que o critério era logicamente fraco já que o curso de qualquer doença também dependia de condições fortuitas, como a gravidade da lesão e a resistência do hospedeiro, nenhum dos quais poderiam ser considerados aspectos "essenciais" da doença. Jolly era um aderente estrito do modelo anatomopatológico estabelecido, segundo o qual uma classificação de doenças tinha de ser baseada em critérios anatômicos (Lopez-Piñero, 1983).

Em princípio, Kraepelin teria concordado com essa visão, já que nesta fase de seu trabalho ele ainda entretinha fortes crenças na possibilidade de identificar mecanismos patológicos (Hoff, 1985). Na prática, porém, ele usou o diagnóstico de uma forma "funcional" e adiou a sua esperança de encontrar uma ligação entre a anatomia e o quadro clínico. Ele insistiu, no entanto, em seu objetivo de curto prazo de encontrar uma classificação válida. Para fazer isso ele teve que mudar sua visão sobre a essência do diagnóstico, ou seja, que não era uma estrada real para a anatomia, mas apenas para o prognóstico. Aschaffenburg, principal colaborador de Kraepelin em Heidelberg, descreveu a mudança do ponto de vista de Kraepelin epigramaticamente: "um diagnóstico errado não é um diagnóstico incorreto, mas um que 'não tem propósito', isto é, não capacita o médico para fazer qualquer palpite adequado quanto ao futuro de seu paciente". 


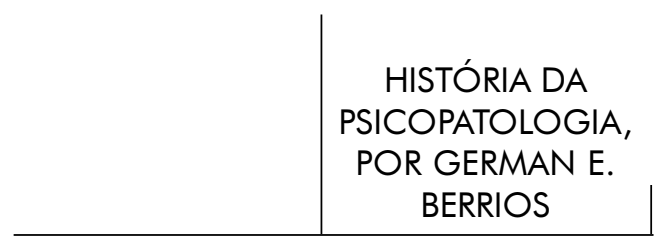

Com relação a isto, baseado em uma versão traduzida da sétima edição, um autor sugeriu que Kraepelin teria insistido em uma "correlação ponto a ponto entre a etiologia, a patologia e a sintomatologia “(Rieder, 1974, p. 199). É evidente que a tradução livre feita por A.R. Diefendorf (Kraepelin, 1907) sobrevaloriza isso. Não há evidência de que uma tal correlação tenha sido seriamente considerada por Kraepelin.

Ele estava talvez preocupado se a abordagem post hoc poderia levar a um cul-de-sac: "Ninguém vai se arrepender mais do que eu se (usando tal abordagem) a demência precoce tornar-se temporariamente uma categoria muito ampla. Assim, ficaria grato em receber sugestões de como dividir este grupo em subtipos clínicos" (Kraepelin, 1897, p. 263), e anos mais tarde, ele escreveu:

Observações futuras de muitos casos dirão se a tentativa de aumentar ou diminuir a "forma" da doença foi justificada. É de se esperar que os limites clínicos dos grupos de doenças desenhados por nós (...) vá mudar no devido tempo. Essas alterações continuarão até que mais conhecimento sobre a correlação das observações através do acúmulo de experiências tenha sido atingido. (Kraepelin, 1919, p. 235)

\section{Sobre o que Kraepelin "realmente disse"}

O que foi dito até agora também ajuda a levar a uma conclusão justa o debate de 1968 em Londres. Contra Fish (1967, 1968), Hoenig (1968) havia afirmado que seria no quadro clínico e não no critério da incurabilidade que a distinção entre as duas principais psicoses estaria baseada. Walk (1968), depois de verificar as fontes, sentiu que estaria do lado deste último. A evidência apresentada em seu artigo indica que, afinal, ambos os lados tinham razão. Há dois aspectos na obra de Kraepelin: o critério metodológico (incurabilidade) a ditar a lógica de sua pesquisa, e o critério clínico (quadro sintomático), resultante da sua pesquisa empírica.

Os dois critérios operavam da seguinte maneira. A Zählkarten (contendo as informações longitudinais) permitia a Kraepelin dividir seu grupo de pacientes de acordo com a incurabilidade e critérios de deterioração. Ele poderia, então, correlacionar dentro de cada grupo a apresentação clínica original e os desfechos. $\mathrm{Na}$ medida em que estava se referindo ao critério metodológico, Fish (1968) estava certo em dizer que Kraepelin "usou o critério da incurabilidade para estabelecer o seu conceito..." (p. 356). Na medida em que estavam se referindo ao critério clínico, Hoenig e Walk estavam certos ao dizer que Kraepelin "apoiou o 
conceito de 'demência precoce' no quadro clínico..." (Walk, p. 644). Mas a história não deveria ter parado aí, pois no artigo publicado mais tarde, intitulado "O aparecimento da insanidade" (Erscheinungsformen des Irreseins), Kraepelin (1920) pôs em questão os seus esforços anteriores, reconhecendo que as sobreposições clínicas relatadas de fato sugeriam a possibilidade de uma psicose unitária. Esta mudança de concepção colocava abaixo a reação de Kraepelin às opiniões céticas de Hoche (Kahn, 1956).

\section{Kraepelin e a psiquiatria francesa do início do século $\mathrm{XX}$}

O debate de 1968 em Londres pode ser considerado como um retrospecto de uma mudança semelhante que teve lugar na França no início do século. Reagindo contra a monografia magistral de Deny \& Roy (1903) que introduziu o conceito de démence précoce de Kraepelin para a psiquiatria francesa, Marandon de Montyel (1905) criticou a visão que a demência precoce sempre levaria a déficits e destacou o fato de que os psiquiatras alemães tinham sido incapazes de identificar um quadro clínico que pudesse caracterizá-la adequadamente ("La démence précoce de M. Kraepelin... n'est ni démence, ni précoce” (p. 247). Infelizmente, Marandon baseou seu argumento em um grande faux pas estatístico. Este foi assinalado por Mongeri (1905) que, em sua resposta, apontou que Kraepelin havia fornecido um quadro clínico e, portanto, não baseara sua classificação apenas no desfecho. Ele também criticou a afirmação de Marandon de que a doença de Kraepelin era "idêntica" à folie dégénérative de Magnan. Curiosamente, Kraepelin foi um convidado de Mongeri (um psiquiatra treinado na França) durante suas duas visitas a Constantinopla (Kraepelin, 1983).

Victor Parant (1905) também criticou Kraepelin, em virtude da sinuosidade dos seus quadros clínicos sugeridos.

As opiniões apresentadas sob o nome de demência precoce são artificiais, sem fundamento e sem confirmação tanto do ponto de vista semiológico quanto anatomopatológico. Elas coletam arbitrariamente estados clínicos independentes e são contrárias à experiência clínica (...) (pp. 240-241)

Coube a Monod (1905) e mais tarde a Mairet \& Margarot (1920) apresentar os pontos de vista de Kraepelin de uma forma menos preconceituosa. Não obstante estes esforços, o conceito de esquizofrenia permaneceu parcialmente incompatível com os pontos de vista originalmente franceses sobre as psicoses crônicas (Pichot, 1982, 1984). 
HISTÓRIA DA

PSICOPATOLOGIA,

POR GERMAN E.

BERRIOS

Discussão e conclusões

A classificação desenvolvida por Kraepelin durante esses anos cruciais (1886-1896) revelou-se frutífera. Sua intenção era identificar um quadro clínico e criar uma taxonomia para as psicoses. Sua análise longitudinal permitiu-lhe utilizar os dados do desfecho como discriminador de sintomas. A análise estatística de Kraepelin, embora simples, gerou uma visão da doença que era anos luz à frente da de Esquirol, cuja técnica de contagem individual só tinha servido para reforçar sua crença em uma visão ontológica da doença (Esquirol, 1838; Berrios, 1988), e também de Griesinger, que havia postulado uma forma de psicoses unitárias (Rennert, 1968). Será sempre motivo de admiração saber quão longe Kraepelin ainda teria ido, se ele tivesse aplicado técnicas estatísticas mais complexas para seus dados. Nessa época Galton já havia sugerido formas de melhorar a análise de correlação, mas é improvável que Kraepelin tenha sabido delas, embora ele tenha conhecido Galton brevemente durante uma de suas viagens a Londres (Kraepelin, 1983, p. 62). Ele só pensou seriamente em empregar um estatístico durante o planejamento do Instituto de Munique, em 1919 (Kraepelin, 1983, p. 218).

Ao tentar acabar com os enviesamentos sistemáticos, Kraepelin também deu um novo significado à noção de confiabilidade e tornou possível o desenvolvimento das bases de dados clínicos. Tanto assim, que os próprios grupos de pacientes que ele ajudou a constituir iriam fornecer os meios para encontrar evidência empírica contra os seus próprios pontos de vista teóricos. Isto é precisamente o que aconteceu no caso de Lange que, utilizando o banco de dados de Kraepelin, foi capaz de desafiar o prognóstico negativo e positivo que ele havia ligado à demência precoce e à insanidade maníaco-depressiva, respectivamente (Vaillant, 1964).

A contribuição de Kraepelin para o desenvolvimento do conceito de psicose é inegável. Ele viveu durante um período de empirismo desenfreado e não desperdiçou as muitas oportunidades clínicas que ele teve à sua disposição. $\mathrm{O}$ conceito de psicose só poderia se cristalizar na confluência de quatro grandes dicotomias: psicoses-neuroses, unitário-múltiplo, orgânico-funcional e exógeno-endógeno (Berrios, 1987b). O trabalho de Kraepelin tinha algo a dizer sobre cada uma delas. Sua mente categórica tinha uma inclinação verdadeiramente kantiana (Dorner, 1969) que ele provavelmente herdara de Wundt (Klein, 1970; Mischel, 1970; Leary, 1978). Por isso, ele foi capaz de impor uma estrutura categórica duradoura sobre a nosologia psiquiátrica, e reforçar isso empiricamente. Este artigo descreveu o desenvolvimento inicial das ideias de Kraepelin, e o contexto metodológico e histórico em que isso ocorreu. Começando com as perguntas que 
Kraepelin fez a si mesmo, em 1886, mostramos como ele chegou a respondê-las dez anos depois. A certeza clínica com a qual ele falou em 1896 foi fundada na análise de uma coorte ampla de pacientes baseada em um sistema de fichas de acompanhamento. Como os seus pensamentos progrediram a partir de 1913 será o tema de outro artigo.

\section{Referências}

Alexander, F. G. \& Selesnick, S. T. (1966). The History of Psychiatry. New York: Harper and Row.

Anonymous (1878). Esthonia. In Encyclopaedia Britannica (Ninth edition, v. VIII, pp. 561-563). Edinburgh: Adam and Charles Black.

Avenarius, R. (1979). Emil Kraepelin, seine Persönlichkeit und seine Konzeption. In Psychopathologie als Grundlagenwissenschaft (ed. W. Janzarik, pp. 62-73). Stuttgart: Enke.

Berrios, G. E. (1985). Positive and negative symptoms and Jackson. Archives of General Psychiatry, 42, 95-97.

Berrios, G. E. (1987a). Dementia during the seventeenth and eighteenth centuries: a conceptual history. Psychological Medicine, 17, 829-837.

Berrios, G. E. (1987b). Outcome prediction and treatment response in schizophrenia. Practical Reviews in Psychiatry, 2, 7-9.

Berrios, G. E. (1987c). Historical aspects of psychoses: nineteenth-century issues. British Medical Bulletin, 43, 484-498.

Berrios, G. E. (1988). The historical development of abnormal psychology. In: E. Miller \& P. J. Cooper. Textbook of Abnormal Psychology. London: Churchill

Livingstone.Blashfield, R. K. (1982). Feighner et al., invisible colleges, and the Matthew effect. Schizophrenia Bullelin, 9, 1-12.

Blashfield, R. K. (1984). The Classification of Psychopathology. Neo-Kraepelinian and Quantitative Approaches. New York: Plenum Press.

Braceland, F. J. (1956). Kraepelin, his system and his influence. American Journal of Psychiatry, 113, 871-874.

Bruch, H. (1969). One hundred years of psychiatry (Kraepelin), fifty years later. Archives of General Psychiatry, 21, 257-258.

Craig, G. A. (1978). Germany 1866-1945. Oxford: Clarendon Press.

Daraszkiewicz, L. (1892). Über Hebephrenie, insbesondere deren schwere Form. Dissertation, Laakmans, Dorpat.

Decker, H. A. (1977). Freud in Germany. New York: International Universities Press.

Del Pistoia, L. (1971). Le probléme de la temporalité dans la psychiatrie française classique. L' Evolution Psychiatrique, 36, 445-474. 


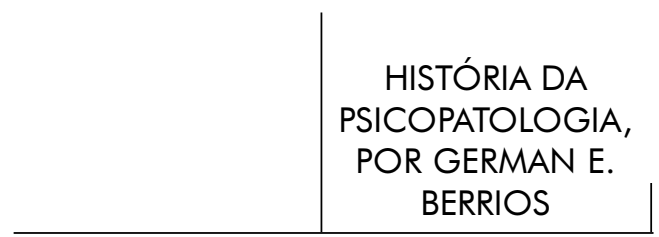

Deny, G. \& Roy, P. (1903). La Démence Précoce. Paris: Baillière et fils.

Dörner, K. (1969). Bürger und Irre. Frankfurt am Main: Europäische Verlagsanstalt.

Ellenberger, H. F. (1970). The Discovery of the Unconscious. The History and Evolution of Dynamic Psychiatry. London: Allen Lane.

Esquirol, E. (1838). Des Maladies Mentales (v. 1 and 2). Paris: Baillière.

Fish, F. (1967). Recent developments in schizophrenia: a review. British Journal of Psychiatry, 113, 1321.

Fish, F. (1968). Kraepelin's nosology. British Journal of Psychiatry, 114, 356.

Grayson, D. A. (1987). Can categorical and dimensional views of psychiatric illness be distinguished? British Journal of Psychiatry, 151, 355-361.

Havens, L. L. (1956). Emil Kraepelin. Journal of Nervous and Mental Diseases, 141, 16-28. Hoenig, J. (1968). Kraepelin and his approach to nosology. British Journal of Psychiatry, 114, 125-126.

Hoenig, J. (1983). The concept of schizophrenia: Kraepelin, Bleuler, Schneider. British Journal of Psychiatry, 142, 547-556.

Hoff, P. (1985). Zum Krankheitsbegriff bei Emil Kraepelin. Nervenarzl, 56, 508-513.

Janzarik, W. (1979). 100 Jahre Heidelberger Psychiatrie. In Psychopathologie als Grundlagenwissenschaft (ed. W. Janzarik, pp. 1-18). Stuttgart: Enke.

Jaspers, K. (1963). General Psychopathology (J. Hoenig and M. Hamilton, trad.). Manchester: Manchester University Press.

Kahlbaum, K. (1863). Die Gruppirung der Psychischen Krankheiten und die Eintheilung der Seelenstörungen. Danzig: A. W. Kafemann.

Kahn, E. (1956). Emil Kraepelin. American Journal of Psychiatry, 113, 289-294.

Klein, D. B. (1970). A History of Scientific Psychology. London: Routledge \& Kegan Paul.

Kolle, K. (1957). Kraepelin und Freud. Beitrag zur Neuren Geschichte der Psychiatrie. Stuttgart: Georg Thieme.

Kraepelin, E. (1887). Die Richtungen der Psychiatrischen Forschung. Vortrag, gehalten hei der Übernahme des Lehramtes an der Kaiserlichen Universität. Leipzig: Dorpat Vogel.

Kraepelin, E. (1893). Lehrbuch der Psychiatrie 4. Auflage. Leipzig: Abel Meixner.

Kraepelin, E. (1894). Die Abgrenzung der Paranoia. Allgemeine Zeitschrift für Psychiatrie, 50, 1080-1081.

Kraepelin, E. (1896a). Über Remissionen bei Katatonie. Allgemeine Zeitschrift für Psychiatrie, 52, 1126-1127.

Kraepelin, E. (1896b). Lehrbuch der Psychiatrie. 5. Auflage. Leipzig: Barth.

Kraepelin, E. (1897). Ziele und Wege der klinischen Psychiatrie. Allgemeine Zeitschrift für Psychiatrie, 53, 840-848.

Kraepelin. E. (1907). Textbook of Psychiatry (7th ed., A. R. Diefendorf , trad.). London: Macmillan.

Kraepelin, E. (1918). Hundert Jahre Psychiatrie. Ein Beitrag zur Geschichte menschlicher Gesittung. Zeitschrift der Neurologie, 38, 161-275. 
Kraepelin, E. (1919). Die Erforschung psychischer Krankheitsformen. Zeitschrift für die gesammte Neurologie und Psychiatrie, 51, 224-246.

Kraepelin, E. (1920). Die Erscheinungsformen des Irreseins, Zeitschrift für die gesammte Neurologie und Psychiatrie, 62, 1-29.

Kraepelin, E. (1921). Bismarcks Persönlichkeit. Ungedruckte persönliche Erinnerungen. Süddetischen Monatshefte, 19, 105-122.

Kraepelin, E. (1983). Lebenserinnerungen. Berlin: Springer.

Lange, J. (1926). Emil Kraepelin. Nature wissenschaften, 14, 1255-1256.

Lanteri-Laura, G. (1972). La chronicité dans la psychiatrie moderne française. Annales (Paris), 3, 548-568.

Lanteri-Laura, G. (1986). Acuité et pathologie mentale. L'Evolution Psychiatrique, 51, 403-416.

Leader (1968). Emil Kraepelin (1856-1926): psychiatric nosographer. Journal of the American Medical Association, 203, 978-979.

Leary, D. E. (1978). The philosophical development of the conception of psychology in Germany, 1780-1850. Journal of the History of the Behavioural Sciences, 14, 113-121.

Lopez-Piñero, J. M. (1983). Historical Origins of the Concept of Neurosis (D. Berrios, trad.). Cambridge: Cambridge University Press.

Mairet, A. \& Margot, J. (1920). La démence précoce. Paris: Dubois.

Marandon de Montyel, E. (1905). Les formes de la démence précoce. Annales Medico-Psychologiques, 2, 246-260.

Mayer, W. (1956). Emil Kraepelin. American Journal of Psychotherapy, 10, 273-276.

Mischel, T. (1970). Wundt and the conceptual foundations of psychology. Philosophy and Phenomenological Research, 31, 1-26.

Mongeri, 1. (1905). Les formes de la démence précoce. Annales Médico-Psychologiques, 2, 261-265.

Monod, G. (1905). Les formes frustrées de la Démence Précoce. Paris: Rousset.

Parant, V. (1905). D'une prétendue entité morbide dite démence précoce. Annales Medico-Psychologiques, 1, 229-241.

Pauly, P. J. (1983). The political structure of the brain; cerebral localization on Bismarckian Germany. International Journal of Neuroscience, 21, 145-149.

Pichot, P. (1982). The diagnosis and classification of mental disorders in Frenchspeaking countries: background, current view and comparisons with other nomenclatures. Psychological Medicine, 12, 475-492.

Pichot, P. (1984). A Century of Psychiatry. Paris: Dacosta.

Rennert, H. (1968). Wilhelm Griesinger und die Einheitpsychose. Wissenschaftliche Zeitschrift der Humboldl Universität, 17, 15-16.

Rieder, R.O. (1974). The origins of our confusion about schizophrenia. Psychiatry, 37, 197-208.

Vaillant, G. E. (1964). An historical review of the remitting schizophrenias. Journal of Nervous, and Mental Diseases, 138, 48-56. 


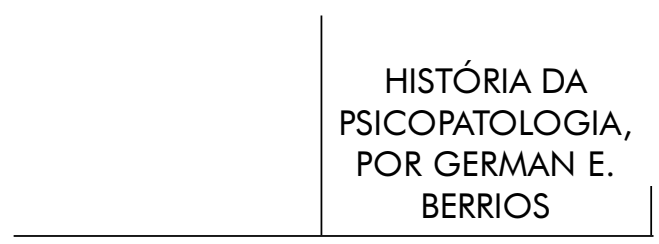

Walk, A. (1968). What Kraepelin really said. British Journal of Psychiatry, 114, 643644.

Wender, P. H. (1963). Dementia Praecox: the development of the concept. American Journal of Psychiatry, 119, 1143-1151.

Wirth, W. (1927). Emil Kraepelin zum Gedächtnis. Archiv gesammte Psychologie, 58, $1-32$.

Zilboorg, G. (1941). A History of Medical Psychology. New York: Norton.

Zubin, J., Oppenheimer, G. \& Neugebauer, G. (1985). Degeneration theory and the stigma of schizophrenia. Biological Psychiatry, 20, 1145-1148.

Zubin, J. (1987). Foreword. In Emil Kraepelin Memoirs. Berlin: Springer

\section{Resumos}

(The first development of Kraepelin's ideas on classification: a conceptual history)

Psychiatry is still living in Kraepelin's world. With this, studies on his writings are a-historical. The selective exegesis of the numerous editions of his book led to a rigid understanding of his contribution. But Kraepelin lived and wrote during an important period in European intellectual history and his writings can only be understood from this perspective. This article analyzes the development of his opinions in terms of the "program of studies" that he planned early in his life, the objective of which was to establish a stable description and classification of the psychoses. He eventually achieved this by longitudinally studying cohorts of patients using methodological criteria such as the course of psychosis and its incurability. In the case at hand, this methodology allowed him to use correlation to identify "clinical pictures" that both represented the "essence" of the disease and provided a taxonomic criterion. Although declaredly a-theoretical, Kraepelin (influenced by Kahlbaum and Wundt) thus managed to construct an empirical support for his Kantian categorization of the psychoses. A discussion on the cultural variables that forged these ideas is included here.

Key words: Mental Disorders - history, Mental Disorders - classification; Psychiatry history, Emil Kraepelin

(Le développement précoce des idées de Kraepelin sur la classification: une histoire conceptuelle)

La psychiatrie vit encore dans un monde kraepelinien et les études sur le travail de Kraepelin sont donc non-historiques. L'exégèse sélective des diverses éditions de son livre a conduit à une conception rigide de sa contribution. Cependant, Kraepelin a vécu et a écrit pendant une période importante de l'histoire intellectuelle européenne et son cuvre ne peut être comprise que dans ce contexte. Cet article analyse le développement 
de ses points de vue en termes de "Programme de Recherches» qu'il avait établi au début de sa vie et dont le but était de créer une description stable et la classification des psychoses. Kraepelin a atteint son but à travers une étude longitudinale de cohortes de patients en termes de critères méthodologiques, comme le cours de la maladie et l'incurabilité. Cette méthode lui a permis d'identifier, par corrélation, des «cadres clinique» qui ne représentaient non seulement «l'essence» de la maladie, mais qui fournissaient aussi un critère taxonomique. Bien que, selon son propre aveu, athéorique, Kraepelin (influencé par Kahlbaum et Wundt) a réussi à construire, de cette façon, un support empirique pour sa catégorisation kantienne des psychoses. Cet article contient d'ailleurs une discussion sur les variables culturelles qui ont façonnées ces idées.

Mots clés: Mental Disorders - histoire, Mental Disorders - classification, psychiatrie histoire, Emil Kraepelin

(El desarrollo inicial de las ideas de Kraepelin sobre la clasificación: una historia conceptual)

La psiquiatria aún vive en un mundo kraepeliano y los estudios sobre la obra de Kraepelin son, consecuentemente, no-históricos. La exegesis selectiva de las varias ediciones de su libro llevaron a una visión rígida sobre su contribución. Sin embargo, Kraepelin vivió y escribió durante un periodo importante de la historia intelectual de Europa y su obra solo puede ser comprendida en este contexto. Este artículo analiza el desarrollo de sus opiniones de acuerdo a los términos del "Programa de investición" que él, tempranamente, planeó en su vida y cuyo objetivo era la creación de una descripción estable y una clasificación de las psicosis. Eso, eventualmente, Kraepelin consiguió estudiando longitudinalmente un conjunto de pacientes según criterios metodológicos, como el curso de la enfermedad y de la incurabilidad. En ese caso, esta metodlogia le permitió identificar por correlación "cuadros clínicos" que representaban la "esencia" de la enfermedad así como ofrecían un criterio taxonónmico. A pesar de claramente a-teórico, Kraepelin consiguió de este modo construir (influenciado por Kahlbaum y Wundt) una base empírica para su categorización kantiana de las psicosis. Una discusión sobre las variables culturales que modelaron esas ideas está incluída.

Palabras clave: Trastornos mentales - historia, Trastornos mentales - classificación, psiquiatría - historia, Emil Kraepelin

(Die anfängliche Entwicklung des Denkens von Kraepelin zur Klassifizierung: eine konzeptionelle Geschichte)

Die Psychiatrie lebt noch in einer kraeppelinschen Welt und die Studien zum Werk von Kraepelin sind folglich nicht historisch. Die selektive Exegese der verschiedenen Ausgaben seines Buches führten zu einer engen Sichtweise seines Beitrages. Doch 
HISTÓRIA DA

PSICOPATOLOGIA,

POR GERMAN E.

BERRIOS

Kraepelin hat in einer bedeutsamen Phase der intellektuellen Geschichte Europas gelebt und geschrieben und sein Werk kann nur in diesem Kontext verstanden werden. Dieser Beitrag untersucht die Entwicklung seiner Ansichten bezüglich des „Forschungsprogrammes”, das er in jungem Alter geplant hat und dessen Ziel die Kreierung einer stabilen Beschreibung und eine Klassifizierung der Psychosen war. Dies erreichte Kraepelin indem er die Veränderungen eines Krankheitsbildes im Laufe der Zeit von Patientengruppen im Sinne von methodologischen Kriterien untersuchte, wie den Verlauf einer Krankheit und die Unheilbarkeit. In diesem Fall erlaubte ihm diese Methodologie, ,klinische Bilder” durch Korrelation zu identifizieren, die sowohl die „Essenz” der Krankheit darstellten wie auch taxonomische Kriterien boten. Obwohl Kraepelin erklärter Nicht-Theoretiker war, hat er es damit geschaff, eine empirische Grundlage für seine Kant'sche Kategorisierung der Psychosen aufzustellen (beeinflusst von Kahlbaum und Wundt). Eine Diskussion über die kulturellen Variablen?, die diese Ideen geformt haben ist dabei.

Schlüsselwörter: Geistesstörungen - Gescichte, Geistesstörungen - Klassifizierung, Psychiatrie - Geschichte

Citação/Citation: Berrios, G. E. (2012 dezembro). O desenvolvimento inicial das ideias de Kraepelin sobre classificação: uma história conceitual. Revista Latinoamericana de Psicopatologia Fundamental, 16(1), 126-146

Editor do artigo/Editor: Prof. Dr. Manoel Tosta Berlinck

Recebido/Received: 15.11.2011 / 11.15.2011 Aceito/Accepted: 5.01.2012 /01.5.2012

Copyright: () 2009 Associação Universitária de Pesquisa em Psicopatologia Fundamental/ University Association for Research in Fundamental Psychopathology. Este é um artigo de livre acesso, que permite uso irrestrito, distribuição e reprodução em qualquer meio, desde que o autor e a fonte sejam citados / This is an open-access article, which permits unrestricted use, distribution, and reproduction in any medium, provided the original author and source are credited.

Financiamento/Funding: Os autores declaram não ter sido financiados ou apoiados / The authors have no support or funding to report.

Conflito de interesses/Conflict of interest: Os autores declaram que não há conflito de interesses / The authors declare that has no conflict of interest. 


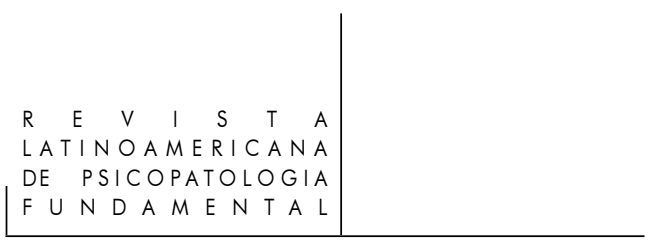

\section{GERMAN E. BERrios}

Médico e filósofo pela Universidad Nacional de San Marcos, Lima, Peru; Psiquiatra; Neurologista; Psicólogo; Filósofo; Historiador e Filósofo da ciência (Oxford University, England); Professor de Neuropsiquiatria e de Epistemologia da Psiquiatria (University of Cambridge, England), desde 1976; Neuropsiquiatra e Chefe do Departamento de Neuropsiquiatria do Hospital Addenbrooke, University of Cambridge, por 32 anos; Coordenador do Comitê de Ética em Pesquisa com Humanos na mesma universidade, por vinte anos; Editor Responsável de History of Psychiatry; Autor de 14 livros, incluindo The History of Mental Symptoms, Descriptive Psychopathology since 19th Century (Prêmio Nacional BMA, 1997), A History of Clinical Psychiatry (com Roy Porter), e Delirio (com F. Fuentenebro) e mais de 400 artigos e capítulos de livros; Membro do Royal College of Psychiatrists; da Associação Britânica de Psicologia e da Academia Britânica de Ciências Médicas; Membro Vitalício do Robinson College, Cambridge; doutor Honoris Causa da Universidade de Heidelberg (Alemanha), da Universidade Nacional Mayor de San Marcos (Peru) e da Universidad Autónoma de Barcelona (Espanha); Grão Oficial da Ordem del Sol (Condecoração do Governo Peruano, 2007); prêmio Ramon y Cajal 2008 concedido pela Asociación Internacional de Neuropsiquiatria.

University of Cambridge

Box 189, Hills Road

Cambridge,

UK CB2 2QQ

e-mail: geb11@cam.ac.uk 